\title{
P2P Web Service Based System for Supporting Decision-Making in Cellular Manufacturing Scheduling
}

\author{
Chapter · August 2013
}

DOI: 10.1007/978-94-007-4722-7_15

\section{CITATION}

1

3 authors, including:

\section{Leonilde Rocha Varela}

University of Minho

153 PUBLICATIONS 405 CITATIONS

SEE PROFILE
READS

21

Susana Costa

University of Minho

4 PUBLICATIONS 2 CITATIONS

SEE PROFILE

Some of the authors of this publication are also working on these related projects:

Project

Project
2. UES: Ubiquitous Oriented Embedded Systems for Globally Distributed Factories of Manufacturing Enterprises View project

4. Development of new generation of crane cabins as integrated visual systems for detection and interpretation of environment View project 


\title{
P2P Web Service based System for Supporting \\ Decision-making in Cellular Manufacturing Scheduling
}

\author{
Maria Leonilde Rocha Varela \\ Departamento de Produção e Sistemas \\ Universidade do Minho \\ Campus de Azurém, Guimarães, Portugal \\ leonilde@dps.uminho.pt \\ Rui Barbosa (a) \\ Susana Costa (b) \\ Msc (a) and PhD (b) students on Engineering and Industrial Management \\ Universidade do Minho \\ Campus de Azurém, Guimarães, Portugal \\ (a) rui_barbosa_1980@hotmail.com
}

(b) surp@gmail.com

\begin{abstract}
With the increase of the Internet and Virtual Enterprises (VEs), interfaces for web systems and automated services are becoming an emergent necessity. In this paper we propose a Peer-to-peer (P2P) web-based decision-support system for enabling access to different manufacturing scheduling methods, which can be remotely available and accessible from a distributed knowledge base. The XML-based modeling and communication is applied to manufacturing scheduling. Therefore, manufacturing scheduling problems and methods are modeled using XML. The proposed P2P webbased system works as web services, under the SOAP protocol. The system's distributed knowledge base enables sharing information about scheduling problems and corresponding solving methods in a widened search space, through a scheduling community, integrating a VE. Running several methods enables different results for a given problem, consequently, contributing for a better decision-making. An important aspect is that this knowledge base can be easily and continuously updated by any contributor through the VE. Moreover, through this system once suitable available methods, for a given problem, are identified, it enables running one or more of them, for enabling a better manufacturing scheduling support, enhanced though incorporated fuzzy decision-making procedures.
\end{abstract}

Keywords- Cellular Manufacturing Scheduling, Web Services, Distributed Knowledge Base, Virtual Enterprise, and Fuzzy Decision-making.

\section{INTRODUCTION}

In today's knowledge-based economy, the competitiveness of enterprises and the quality of work life are directly tied to the ability of effective creation and share of knowledge both, within and across organizations.

Manufacturing scheduling is a complex task that involves a wide range of knowledge. Slight differences on the manufacturing environment originate distinct problems, which even though being closely related, require different solving methods to be applied.
The effective and efficient resolution of those problems begins with the identification of suitable scheduling methods for solving them. When there are alternative methods for solving a problem we can obtain alternative solutions, which should be evaluated against specified criteria or objectives to be reached. Thus, users are able to properly solve a problem, through the execution of one or more scheduling methods, local or remotely accessible through the web, and, subsequently, select de most suited solution obtained.

This work attempts to offer new possibilities to scheduling, giving a contribution in terms of manufacturing scheduling problems solving through a P2P web-based decision support system. The system follows a networkedbased computing model, which permits sharing scheduling knowledge by means of a distributed knowledge base. This distributed scheduling repository enables accessing knowledge arising from an extended range of contributors and, therefore, a potentially more useful search, through a widened search space, based on the principles of Virtual Enterprises (VE) [1, 2].

The system permits the characterization of each problem to be solved and, then, the access to corresponding solving methods. For problems identification, a problem classification model that includes a set of parameters is used. The data representation model for scheduling problems and related concepts is based on XML (extensible markup language), used as a specification language for scheduling data representation and processing on the web $[3,4,5]$.

Moreover, fuzzy decision-making procedures are incorporated in the proposed $\mathrm{P} 2 \mathrm{P}$ web-based scheduling decision-support system, in order to enable a better manufacturing scheduling activity, by the users, namely regarding cellular manufacturing problems solving.

This paper is organized as follows. The next section briefly presents the state of the art, regarding web-based scheduling approaches. Section 3 describes the nature of cellular manufacturing scheduling. Section 4 presents the proposed P2P web-based system, including a brief 
description of its architecture and main functionalities. Moreover, an example is given to illustrate the system's use, for solving a cellular manufacturing scheduling problem. Finally, in section 5, a conclusion is presented.

\section{STATE OF THE ART}

Recently we notice a tendency for an increase in scheduling systems accessible through the Internet. The systems that we came across usually involve solvers or a set of solvers, addressing each one the resolution of a restricted range of scheduling problems, using specific techniques or tools, such as mathematical programming. Moreover, they are not usually designed to easily incorporate new methods' implementations by users.

An example of a web system that can be used for scheduling purposed is the NEOS Server, developed under the auspices of the Optimization Technology Center of Northwestern University and Argonne National Laboratory, generally used for optimization problems solving. It makes nearly 50 solvers available through a broad variety of network-based interfaces. According to the authors, although having evolved along with the web and the Internet, it is limited to some degree by early design decisions (http://www-neos.mcs.anl.gov/).

We can also refer the BBN's Vishnu scheduling system, as another interesting web-based scheduling system (http://vishnu.bbn.com) and the FortMP, a Mathematical Programming Solver, by Mitra's Group, at Brunel University (http://www.brunel.ac.uk/depts/ma/research/com).

The e-OCEA, a portal for scheduling intends to support identifying scheduling problems, to help development of new algorithms and to conduct benchmarks through the Internet. However, one requirement of this system is to only consider elements (algorithms, data sets, schedules and modules) that are e-OCEA compatible ones (http://www.ocea.li.univ-tours.fr/eocea).

The LekiNET, a prototype Internet scheduling environment, by Benjamin P. C. et al. (http://www.stern.nyu.edu/om/software/lekin/index.htm), which has migrated from LEKIN, a flexible job shop system, is a system that has some similarities to our proposed system, but focusing more on cost effective choices, based on scheduling agents for problems solving. The authors propose a migration scheme to transform existing standalone scheduling systems to Internet scheduling agents that can communicate with each other and solve problems beyond individual capabilities. They treat each system as an agent and build the relations between the systems. So, wrappers need to be specifically designed for each system $[6,7]$.

\section{Cellular ManufaCtURINGS SCHEDUling}

Manufacturing scheduling consists on a temporal assignment of tasks to manufacturing resources, where one or more goals and constraints have to be considered. It is one of the significant tasks to be performed to achieve competitive manufacturing levels, which usually means to deliver products on time and to use resources efficiently.

Moreover, low flow times, few ongoing jobs in the system, low inventory levels, high resource utilization and, certainly, low production costs are also important objectives, which can be better satisfied through the execution of the most suitable scheduling methods available for solving each particular problem.

In order to perform the scheduling process it becomes necessary to clearly specify the problem to be solved. Manufacturing scheduling problems have a set of characteristics that must be clearly defined.

Due to the existence of a great variety of scheduling problems there is a need for a formal and systematic manner of problem representation that can serve as a basis for their classification. A manufacturing scheduling problems notation for achieving this was developed by Varela, et al. $[8,9]$, based on existing notations available in the literature [10 - 13]. This notation allows identifying the characteristics of each problem to be solved, and it is used as a basis for an XML-based problem specification model used in this work.

After clearly specifying a manufacturing scheduling problem it turns necessary to select a suitable method for solving it. Among the variety of scheduling methods available it is possible to identify three fundamental categories [14]:

- Calculus-based (analytical, based on derivatives);

- $\quad$ Enumerative (brute-force);

- Heuristic guided random search (e.g. SA, Genetic Algorithms and Neighborhood search approaches).

The first two categories deal, mainly, with algorithms that are not appropriate for solving problems of great dimension or lack analytical structure because of the computational time to find a solution [14, 15 and 16].

The third category includes heuristic and approximate algorithms [17, 18 and 19] that are more appropriate for the solution of NP-hard or NP-complete, known as "difficult" problems [10 and 11]. Although these kinds of algorithms do not guarantee optimal solutions, they are usually faster and they allow obtaining near-optimal solutions or usually at least acceptable ones. Sometimes they could even reach an optimal solution. Most of these algorithms are relatively recent and have been increasingly explored [6]. Some wellknown heuristic algorithms refer to Simulated Annealing, Tabu Search and Genetic Algorithms and other are neighborhood search based approaches [6, 10, 11 and 18].

Scheduling methods for solving cellular manufacturing problems have to concerns about ordering jobs to be performed on a set of machines or work centers, in a given manufacturing environment, which is usually characterized by its possibility to process a range of different operations integrating a set of related work, generally grouped together, based on some similarities, which to some extent, enable to consider them to be more or less closely related to each other, in terms of processing requisites. In this context machines or work centers are usually equipped with flexible machines, in order to enable to process some quite different operations, among a set of more or less closely related jobs, usually referred as a family of jobs.

Therefore, in cellular manufacturing usually arise more or less complex manufacturing scenarios, even when processing simple jobs, including only one or two operations and in this sense it may become an even more complex task 
to select appropriate solving methods, for each given manufacturing scheduling problem.

\section{SCHEDUling WEB SERVICES BASED SYSTEM}

In our proposed system any method which is accessible through the Internet, once its signature and location are specified within our web system, can be used, for solving problems put by users. No further requirements are necessary for being able to remotely use available methods. The association of scheduling problems to resolution methods is done using the information available in a DKB, about both problems and solving methods [8]. We did not come across to systems with identical architecture and underlying approach, i.e. oriented for solving a large variety of manufacturing scheduling problems based on a continuously updatable distributed knowledge base, which allows a network of peers to provide scheduling services to users and the dynamic enlargement of the number of methods that can be accessed.

\section{A. General Web Service Description}

A web service enables a client to remotely invoke a method on a given machine, connected through a network, upon receiving a description of a problem instance to be solved, through a selected method, by sending a request with the data for running the method for the given problem instance. Afterwards the results are returned to the client in order to be transformed and presented to the final user in a certain format. Those web services can be identified and described over the web, by using UDDI registry and WSDL descriptions, as shown in Figure 1 (http://www.w3.org).

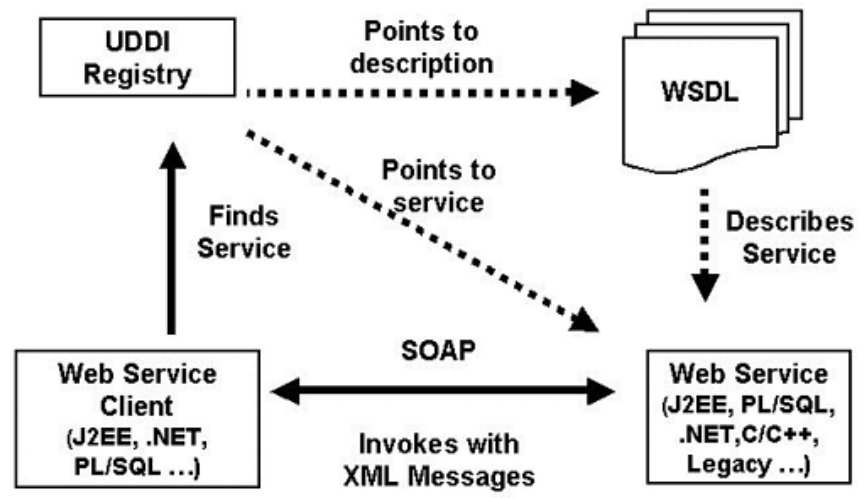

Figure 1. Web service infrastructure.

Moreover, the web services may be implemented and accessible for remote executions by using different platforms, programming languages and communication protocols. Therefore, web services enable to remotely invoke methods, namely manufacturing scheduling ones, which can be performed, for instance, through SOAP (Simple Object Access Protocol, http://www.w3.org/TR/soap/).

\section{B. Web System Architecture}

Web systems are systems that emerged from previous traditional systems, which are systems that beyond other advantages are able to implement web services that can be easily used and shared through the web [20].

Moreover, the last tendencies show that computing environments are characterized by increasing heterogeneity, distribution and cooperation, where distributed knowledge bases also play an important role [20].

In networked environments, distributed knowledge bases and intelligent brokers, for information retrieval from specialized servers and knowledge repositories distributed across the Internet, enable to establish high-quality problem solving, through knowledge and resources sharing, forming virtual enterprises (VE) $[1,2]$.

In VEs several business partners contribute with a local knowledge base (KB) component within networked environments. In these environments each member can be seen as a VE business partner interested on solving manufacturing scheduling problems, as illustrated through the P2P (peer-to-peer) architecture on Figure 2 (http://www.dmst.aueb.gr/dds/pubs/jrnl/2004-ACMCSp2p/html/AS04.html).

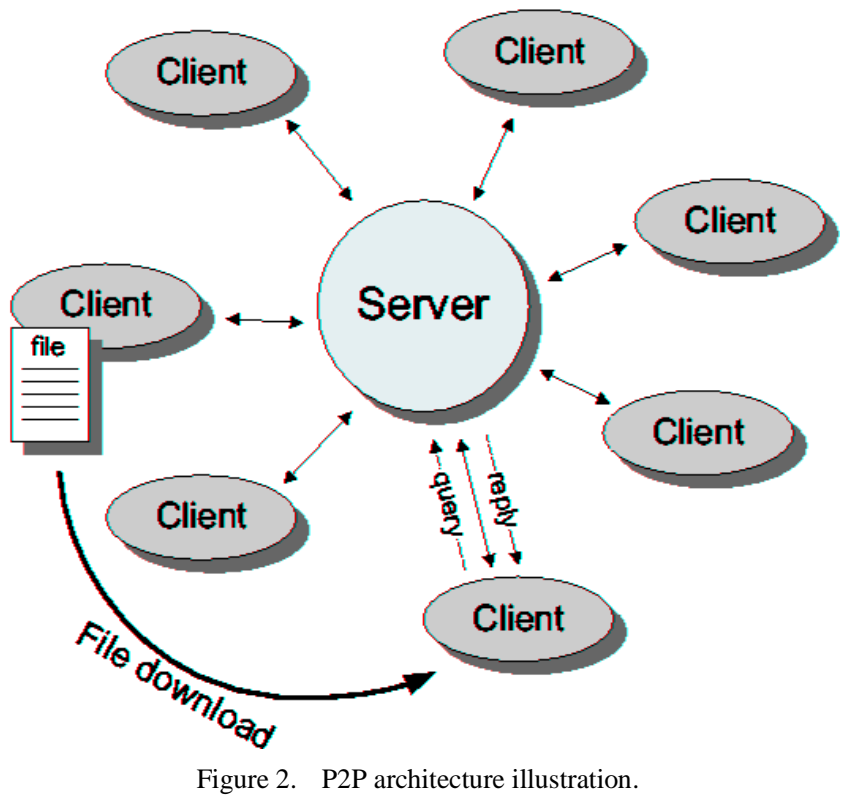

One of the greatest benefits of these networked environments, in the context of this work, is to easily support the concept of community. Consequently, it is possible for users to organize themselves into groups that can collaborate with each other in order to achieve certain goals. One of the main goals aimed at in this work is the collaborative improvement of the resolution of manufacturing scheduling problems. This is possible through the DKB for manufacturing scheduling by accessing several different scheduling approaches. This is achieved by providing a mechanism that allows the members of the organization's network to share their scheduling knowledge and scheduling methods.

Whenever a member stores knowledge in its $\mathrm{KB}$ component, he/ she is automatically contributing to the enrichment of the whole distributed knowledge repository, which is available to all members of the VE. 
Different manufacturing scheduling methods' implementations may provide results in different formats; consequently the system must have a description of them in order to format them according to the problem output to be returned to the client as the very last step of the service.

Moreover, the proposed system integrates an interface, for problem results presentation (as well as for problem data input), which is automatically generated by the system according to the corresponding implemented method's signature, previously specified.

Therefore, for the system to be able to use such implementations in a programmatic way, they must be described within the system. This description must include, among other things, the address to the running method or program and its signature, which, in turn, includes the definition of the parameters that are necessary for its execution (inputs) and its output format.

All the scheduling information is stored in XML documents, which are validated according to associated DTDs, before being put in the corresponding knowledge base component [8 and 20].

The result from running an implemented method on a given problem instance can be delivered to the client as an XML file and/ or can be transformed into some more expressive output, like a Gantt chart or other charts, namely obtained through excel files processing, which is another input and output format enabled through the proposed system, among others.

As previously referred the DKB scheduling system is based on the principles of VE. In this VE, each business partner contributes with a local knowledge base (KB) component in the organization's network. Each one can be seen as a VE member or partner interested on solving scheduling problems. At each business partner the knowledge base can be continuously improved with new problem descriptions and available solving methods.

Whenever a member stores knowledge in its $\mathrm{KB}$ component, he/ she is automatically contributing to the enrichment of the whole distributed knowledge repository, which is available to all members of the VE.

The methods are usually available in the knowledge base of the VE partners but they can also be found in other sites not belonging to the community.

\section{Web System Functionalities}

The system has been designed and implemented as a set of web services (http://www.w3.org) using the SOAP protocol (http://www.w3.org/TR/soap/).

Each web service accepts as input a problem definition, i.e. data for running a method, and returns outputs from a method's invocation.

The SOAP protocol (Simple Object Access Protocol), like other communication protocols, consist on a possible way that can be used for remote methods invocation and can be defined as the sequence and structure of requests and responses required to invoke communications on a remote machine [20, 21].

The XML specification language provides a vocabulary for describing remote procedure calls, which are transmitted between computers using the Hyper Text Transfer Protocol (HTTP). SOAP clients make procedure requests to a server, which returns results to the clients. Clients use the same HTTP facilities as web browser clients, and servers use the same HTTP facilities as web servers. The SOAP protocol requires a minimal number of HTTP headers to be sent along with the XML method request for solving a given problem instance.

Upon receiving a request, a server must deliver a response to the client. The response may take one of two forms: the result of processing the method or a fault report, indicating that something has gone wrong in handling the request from the client. As with a request, the response consists of HTTP headers and an XML payload.

By using the SOAP protocol we are able to invoke scheduling methods implemented on different programming languages. Moreover, these methods, local or remotely available, may be running on different platforms. Figure 3 shows the main system's functionalities of out system.

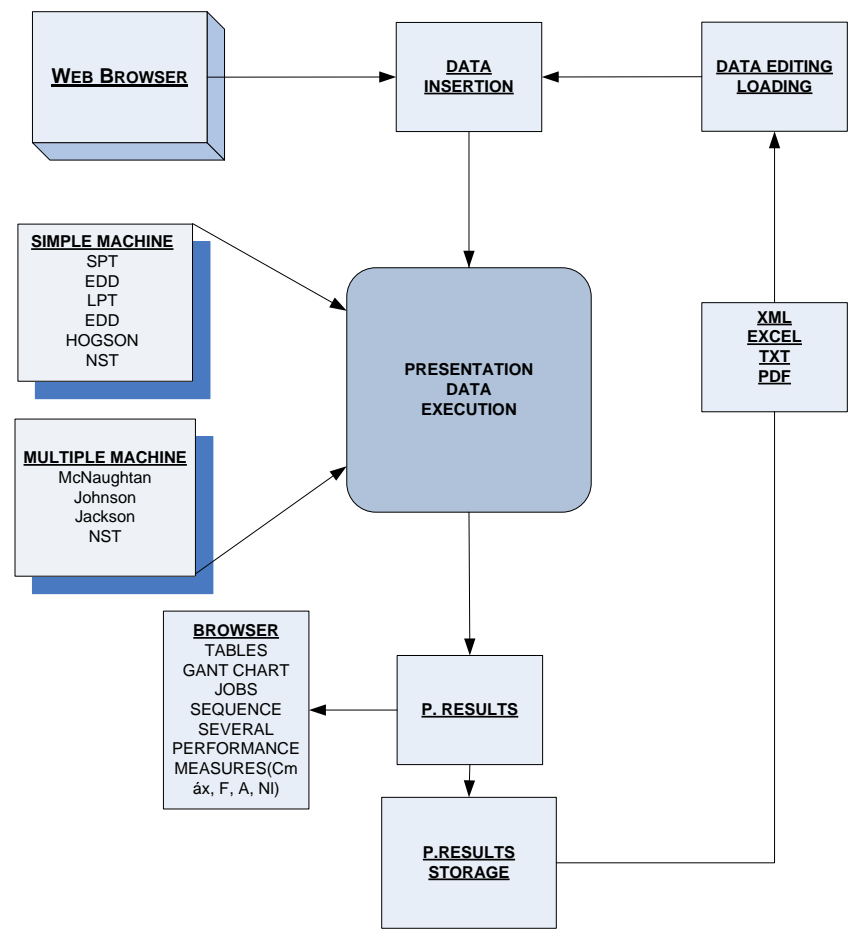

Figure 3. Main web system functionalities.

As referred previously, our web scheduling system includes a Knowledge Base component that encompasses all knowledge and modules necessary for remote methods invocation and for performing a set of other system functionalities. This component is controlled by ASP (Active Server Pages) and corresponding server-side components.

On the other hand, there are also correspondent components for each of the methods servers waiting for requests.

This environment is heterogeneous as servers can use their own technology, i.e. use different implementation languages or/ and different operating systems. 
Several other protocols that could be used exist, namely XML-RPC (eXtensible Markup Language - Remote Procedure Call), CORBA (Common Object Request Broker Architecture) or RMI (Remote Method Invocation) [20, 21].

\section{Fuzzy decision-making}

An important aspect for improving the decision-making process consists on enabling the use of fuzzy parameters on the scheduling problems specification. For this purpose, fuzzy sets are being used.

The main goal of a fuzzy approach is to find the solution or the alternative decisions that may better satisfy and suite to a given situation that occurs in a fuzzy environment [22].

The proposed system can be used as a support tool, for the decision-making process, by providing, in real-time, a range of alternative solutions to the decision maker, indicating the advantages and disadvantages (trade-offs) of each evaluated alternative. With such information the decision maker can take an enlightened choice of the best alternative for each specific application scenario.

Great part of the decision-making process occurs in an environment in which the objectives, the constraints and the consequences of possible actions are not accurately known. To deal quantitatively with imprecision it becomes sometimes necessary to appeal to tools provided by the fuzzy sets theory [23].

With this type of approach we can easily represent any fuzzified parameter of our optimization problem. For instance, we may represent fuzzy coefficients, as for example, "the deadline of a job $\mathrm{x}$ is about 7 time units". This meaning that the preference of the decision maker is 7 time units but deviations are allowed to the left and right of the preferred value, by using an appropriate fuzzy function [22]. Moreover, other situations occur when we do not have access to precise information regarding some other kind of problem data, namely regarding a set of parameters that is necessary to be specified for each problem instance to be solved, according to a nomenclature used [24 and 25], which includes a wide range of parameters related to the manufacturing environment and concrete problem characteristics to be defined.

\section{E. Web System Illustration}

The following image, in Figure 4 illustrates de developed system's interface for supporting the user to solve a given cellular manufacturing scheduling problem.

Though the system's interface the user is able to choose among a number of alternatives ways for problem data insertion, namely by loading a file in xml, excel, pdf or txt format, or by directly data insertion through the web browser interface.

Once the user inserted the problem data a set of alternative methods is put available through the system's interface, for solving the given problem, for single up to multiple machines problem cases, varying from simple sequencing rules or procedures up to more complex methods, including meta-heuristics as, for example, based on neighborhood search techniques.
Moreover, the system enables to apply fuzzy logic to some specific problem parameters, as described above, which also enriches the range of results that can be obtained, for a given problem instance resolution, therefore, enabling a better decision-making, for each specific scenario considered.

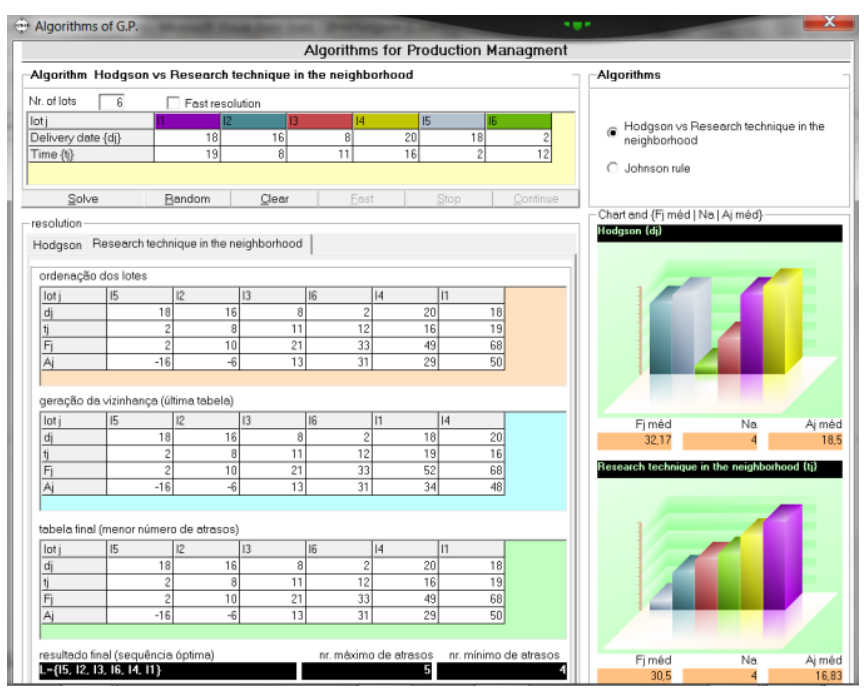

Figure 4. Web system interface illustration.

In terms of problem results, several alternatives are also available through the system, for results presentation as well as for results storage, in xml, excel txt or pdf formats.

Particularly interesting results presentations arise from the use of Gantt chart, tables or other graphics, as shown if Figure 4, which enable even more clear results interpretation.

\section{CONCLUSION}

In today's knowledge-based economy, the competitiveness of enterprises and the quality of work life are directly tied to the ability of effective creation and share of knowledge both, within and across organizations.

In production enterprises, it is important nowadays, as a competitive strategy, to explore and use software applications, now becoming available through the Internet and Intranets, for solving scheduling problems, which can be achieved in an easily way by using web service technology.

This work is based on an XML-based specification framework for manufacturing scheduling concepts modeling, which is used as specification language for better supporting manufacturing scheduling decision-making, based on web services. Some of the important functions of the proposed system include the ability to represent scheduling problems and the identification of appropriate available methods for solving them in the context of a P2P network and underlying distributed knowledge base about scheduling problems and solving methods.

The XML-based data modeling is used in order to make possible flexible communication among different scheduling applications. This modeling and specification language contributes to the improvement of the scheduling process, by allowing an easy selection of several alternative methods 
available for problems solving, as well as an easy maintenance of the knowledge base, by supporting the scheduling web services. This is achieved by providing a user-friendly way of new knowledge insertion, which includes knowledge about scheduling problems and solving methods as well as its implementations, available through the Internet and remotely invoked. These may be implemented on different programming languages and running on different platforms. Such implementations are easily accessible through web services for solving scheduling problems, where cellular manufacturing based ones are of primordial importance, through the invocation of local or remotely available scheduling methods. The system allows comparing different solutions obtained by the running different methods for a same scheduling problem, and to choose the best solution found to solve each problem, according to a given performance measure to be reached.

The XML based specification can be generated and visualized by computers in appropriate and different ways. An important issue is that the data representation model is general, accommodating a large variety of scheduling problems, which may occur in different types of production environments, besides cellular manufacturing systems.

Although the main goal is the service for scheduling problems solving, the system can also be used for teaching purposes, and from this point of view, several different features can be put forward by the system. These include historical and referencing information about each problem class and solving methods. The system will grow as more knowledge is included in the distributed knowledge repository.

The web system is based on an Internet-based infrastructure, associated to a distributed knowledge base (DKB). This DKB is spread through a set of members (partners) forming a virtual enterprise. These members can store information about methods for solving different kinds of manufacturing scheduling problems, as well as its implementations. Each partner, in a decentralized computing environment, is able to directly interact with each other as well as with any other external user. This web-based communication and direct collaboration is enabled through a dynamic web interface, which permits executing diverse scheduling functions, including the ability to represent different scheduling problems, search for appropriate solving methods and running methods that are available and accessible through the DKB, within the VE's network. As running different methods enables obtaining different solutions for a given problem, we believe that the proposed system may contribute to a better decision-making process, enhanced by means of fuzzy based support and collaboration of partners organized as a virtual enterprise.

\section{REFERENCES}

[1] Putnik G. D. (2000b). BM_Virtual Enterprise Architecture Reference Model. Technical Report RT-CESP-GIS-2000-<GP-01>. Universidade do Minho, Portugal.
[2] Putnik, G. D. (2001) BM_Virtual Enterprise Architecture Reference Model, in A. Gunasekaran (Ed.), Agile Manufacturing: 21st Century Manufacturing Strategy (pp. 73-93), Elsevier Science Publ., UK.

[3] Abiteboul, S., et al., 2000. Data on the Web - From Relations to Semistructured Data and XML, Morgan Kaufmann Publishers. USA.

[4] Ceponkus, A., Hoodbhoy, F., 1999. Applied XML, Wiley Computer Publishing. USA.

[5] Harper, F., 2001. XML Standards and Tools, eXcelon Corporation, USA.

[6] Pinedo, M., Scheduling Theory, Algorithms and Systems, PrenticeHall Inc. USA, 2002.

[7] Yen, B. et al. Internet Scheduling Environment with Market-Driven Agents. IEEE Transactions on Systems, Man and Cybernetics - Part A: Systems and Humans, Vol. 34, No. 2, March, 2004.

[8] Varela, L., Aparício, J., Silva, S, 2002a. An XML Knowledge Base System for Scheduling Problems. In IICS'02 - Proceedings of the Innovative Internet Computing System, Kuhlungsborn. Germany. Springer-Verlag in the Lecture Notes in Computer Science series.

[9] Varela, L., Aparício, J., Silva, s., 2002b. Scheduling Problems Modeling with XML. In The 4th International Meeting for Research in Logistics, Lisbon. Portugal. Proceedings of Research in Logistics.

[10] Blazewicz, J., et al., 1996. Scheduling Computer and Manufacturing Processes, Springer-Verlag. Germany.

[11] Brucker, P., 1995. Scheduling Algorithms, Springer-Verlag. Germany.

[12] Graham, R. L., Lawler, E. L., Lenstra, J. K., Rinnooy Kan, A. H. G., 1979. Optimization and Approximation in Deterministic Sequencing and Scheduling: A survey. In Annals of Discrete mathematics.

[13] Jordan, C., 1996. Batching and Scheduling, Springer-Verlag. Germany.

[14] Artiba, A., 1997. Elmaghraby, S., The Planning and Scheduling of Production Systems, Chapman \& Hall. UK.

[15] Conway, R. W., Maxwell, W. L., Miller, L. W., 1967. Theory of Scheduling, Addison-Wesley Publishing Company, Inc. England.

[16] French, S., 1982. Sequencing and Scheduling - An Introduction to Mathematics of the Job-Shop, John Wiley and Sons, Inc.

[17] Arts, E., Lenstra, J. K., 1997. Local Search in Combinatorial Optimization, John Wiely \& Sons.

[18] Morton, T., Pentico, D., 1993. Heuristic Scheduling Systems, John Wiley \& Sons Inc. USA.

[19] Osman, I. H., Kelly, J. P., 1996. Meta-Heuristics: Theory and Aplications, Kluwer Academic Publishers.

[20] Varela, M. L. R., Aparício, J. N., Carmo-Silva, S. (2005). A Scheduling Web Service. In Kendall G., Burke E., Petrovic S., Gendreau M. (Eds.), Multidisciplinary Scheduling - Theory and Applications. Nottingham, UK. Springer. ISBN 0-387-25266-5.

[21] Laurent, S., et al., 2001. Programming Web Services with XML-RPC. O’Reilly \& Associates, Inc.

[22] Varela, M. L. R., Ribeiro R. A., "Evaluation of Simulated Annealing to solve Fuzzy Optimization Problems", Journal of Intelligent and Fuzzy Systems, 2002.

[23] L. Zadeh, Fuzzy Sets, Information and Control, 18 (1965), 338-353.

[24] Varela, M. L. R., Carmo-Silva, S. (2008). An Ontology for a Model of Manufacturing Scheduling Problems to be Solved on the Web. In Azevedo, A. (ed), Innovation in Manufacturing Networks, 8th IFIP International Conference on Information Technology for Balanced Automation Systems (pp. 197-204), Porto, Portugal, June 23-25, 2008, Springer. ISSN: 1571-5736 / 1861-2288 (Internet), ISBN: 9780-387-09491-5; eISBN: 978-0-387-09492-2.

[25] Carmo-Silva, S., Varela, M. L. R. (2011). An Ontology for Production Systems Environments of Scgeduling Problems. In Putnik, G. D., Ávila, P. (eds.), Business Sustainability II, Management Technology and Learning for Individuals, Organisations and Society in Turbulent Environments. University of Minho \& ISEP. 